\title{
Chaotic dynamics in a simple dynamical green ocean plankton model
}

\author{
Roger Cropp* \\ Griffith School of Environment, Griffith University, Nathan, Queensland, 4111, Australia \\ Irene M. Moroz*, John Norbury* \\ Mathematical Institute, University of Oxford, Andrew Wiles Building, ROQ, Woodstock Road, \\ Oxford, OX2 6GG, UK.
}

\begin{abstract}
The exchange of important greenhouse gases between the ocean and atmosphere is influenced by the dynamics of near-surface plankton ecosystems. Marine plankton ecosystems are modified by climate change creating a feedback mechanism that could have significant implications for predicting future climates. The collapse or extinction of a plankton population may push the climate system across a tipping point. Dynamic green ocean models (DGOMs) are currently being developed for inclusion into climate models to predict the future state of the climate. The appropriate complexity of the DGOMs used to represent plankton processes is an ongoing issue, with models tending to become more complex, with more complicated dynamics, and an increasing propensity for chaos.

We consider a relatively simple (four-population) DGOM of phytoplankton, zooplankton, bacteria and zooflagellates where the interacting plankton populations are connected by a single limiting nutrient. Chaotic solutions are possible in this 4dimensional model for plankton population dynamics, as well as in a reduced 3dimensional model, as we vary two of the key mortality parameters. Our results show that chaos is robust to the variation of parameters as well as to the presence of environmental noise, where the attractor of the more complex system is more robust than the attractor of its simplified equivalent. We find robust chaotic dynamics in low trophic order ecological models, suggesting that chaotic dynamics might be ubiquitous in the more complex models, but this is rarely observed in DGOM simulations. The physical equations of DGOMs are well understood and are constrained
\end{abstract}

${ }^{*}$ Corresponding Author: Roger Cropp Tel: +61 737354036

Email addresses: r.cropp@griffith.edu.au (Roger Cropp), Irene.Moroz@maths.ox.ac.uk (Irene M. Moroz), john.norbury@lincoln.ox.ac.uk (John Norbury) 
by conservation principles, but the ecological equations are not well understood, and generally have no explicitly conserved quantities. This work, in the context of the paucity of the empirical and theoretical bases upon which DGOMs are constructed, raises the interesting question of whether DGOMs better represent reality if they include or exclude chaotic dynamics, but also points to a need for a comprehensive approach to model development and testing. We contend that the DGOM community can learn important lessons from the analysis of simple models, and should consider a spectrum of methods from the analysis of simple(r) zero-dimensional models of population interactions through to numerical simulations of fully coupled biogeophysical models.

Keywords: chaos, stiletto attractor, dynamic green ocean model, plankton model; 


\section{Introduction}

The prospect of anthropogenic climate change has generated substantial interest in the role of ocean plankton systems in biogeochemical cycling in the ocean. Plankton may have a significant influence on climate, primarily by drawing down carbon dioxide from the atmosphere and sequestering it in the deep ocean (the 'Biological Pump' [42]), and by producing dimethylsulphide and other volatile compounds that may affect cloud formation over the oceans (the 'CLAW hypothesis' [5]). Dynamic green ocean models (DGOMs) include several plankton functional types (PFTs) in order to resolve the role of plankton in biogeochemical cycling, usually grouped according to their biogeochemical roles rather than their phylogenetic relationships [19]. The number of PFTs required is dependent on the purpose of the model, with Hood et al. [28] noting that the performance of PFT models is more closely related to their tuning than their complexity. Le Quere et al. [33] suggest that at least ten key PFTs are required to resolve climatically important biogeochemical cycling in the oceans. However, there has been significant debate over whether plankton ecosystems are sufficiently well understood to place any reliance on the results of models that include multiple PFTs $[1,32]$.

Pragmatically, the demand to improve the resolution of the role of ocean biology in atmosphere - ocean gas exchange has meant that complex DGOMs are already being used and extended (i.e. [33, 39, 48]). Complex marine plankton models that explicitly resolve the ecological interactions between species, populations and functional types are also being developed for applications in coastal ecology and fisheries management (i.e. [31]). These models are perforce complex, resolving at least one limiting nutrient and often three trophic levels. The value of these models depends on essentially subjective assessments of how well the forms of the equations capture the essence of the population interactions; and of how representative the dynamics of the model in the region of parameter space examined are of the entirety of the realistic parameter space.

Major projects comparing the numerical predictions of these models have been established, with the objectives of evaluating and synthesising understanding to optimise the contributions such models make to the climate change discussion $[3,52]$. A substantial literature documenting these projects has already been published (for example, $[3,52,25,48,21,41,45])$. These model inter-comparison projects, that essentially test the abilities of DGOMs to reproduce observed data, are consistent with the accepted semantic view of theories [50]. In this view, science is the process of continually developing models and comparing them to the real world, so the model inter-comparison projects are part of the scientific process. However, it is easy to be seduced by the sophistication of the models. Their complexity means that assessments of numerical solutions, in the absence of supporting analysis to put the 
numerical results in context and aid their interpretation, is often subjective. It is certain that evaluating the same model in a different part of parameter space, or the same model with a different process functional form, will produce a different simulation result, and it is highly likely that such a result would be substantially different. The numerical investigation of a model, or comparison of the simulation results of two models, provides knowledge for that specific implementation (parameter values, functional forms, etc) of the model. The likelihood of any one implementation of a model being the one that captures reality with veracity is infinitesimally small. The vast solution spaces for these models (see [7] for a discussion of this issue) means that numerical investigations of complex models can at best provide an island of knowledge in a sea of ignorance.

The debate over the merits of adding complexity to the generally successful nutrient, phytoplankton, zooplankton (NPZ) models of the 1990s by resolving plankton functional types identifies a pressing need for better understanding of the behaviour of complex ecological models [34, 35, 40]. Numerous results suggest that increased model complexity generates an increased propensity for chaos [23], making short term prediction difficult and in many cases defeating the purpose of adding complexity to the models. The emphasis of attempts to improve the understanding of, and build confidence in, DGOMs initially focused on increasingly accurate measurements of PFT traits (parameter values) and more accurate and comprehensive data to calibrate and validate the models $[28,33]$, although a recent trend has been to improve the biological realism of the models by making them adaptive, for example [4]. However, the current "industry standard" of considering a model to be useful for prediction if it can both reproduce contemporary data and appear plausible, is concerning to many in the field.

The so-called self-organising models present an interesting case. Self-organising often means that the model includes processes that mimic natural selection (for example $[21,6])$. Natural selection is a very powerful optimising algorithm, as evidenced by the widespread use of Genetic Algorithms to solve computationally difficult optimisation problems [37], so the fact that these models can reproduce spatial and temporal patterns of biology is perhaps not surprising. Follows et al. [21] provide a case in point, where they describe the ability of their model to reproduce global biogeographical fields of microbial communities as an "emergent" property of the model. In this case the deterministic model includes stochastic, natural selection processes that allow the biology to adapt to its environment (or fail to adapt and go extinct), and drive the spatial and temporal distributions of the populations, matching them to the geochemistry. Such knowledge of the properties of the model allows its results to be put into context. Whether this means the models are useful for prediction is moot, as it suggests the rule that the physical environment is essentially determining the biology, and that population interactions are secondary. While the effect of ocean 
physics on the dynamics of marine ecosystems is well-established (for example, [27]), it should not be assumed that the physics will always trump the ecology, and that the endogenous dynamics of the ecosystem equations are of lesser importance. Physical processes may well dominate in the very long term of climate change, but it is likely that population interactions will dominate the response to short-term or more subtle changes, and we should not abandon attempts to understand models that represent population interactions deterministically.

There are many studies of predator/prey and competition systems in population biology/ecology, with a few of these studies considering chaos in higher-dimension models. Chaos in a three-species Lotka-Volterra model was perhaps first demonstrated by Gilpin [24], and in more realistic three-species ecosystem models shown by Hastings and Powell [26], with later work by McCann and Yodzis [36], Deng [14, 15] and Deng and Hines [16, 17]. Cropp and Norbury [11] showed that the population model of Hastings and Powell [26] could be written in an ecologically consistent, mass conserving form with a subtle change that retained the chaotic properties of the model.

We consider the chaotic dynamics of a four-population ecosystem model that is an ecologically consistent, mass conserving system. This extends the earlier work of Cropp and Norbury $[8,10]$ in performing a more systematic analysis of the dynamics of the Moloney et al. [38] model to consider chaotic dynamics in such systems. We investigate the dynamics of a relatively simple, generic model with four plankton populations (PFTs) to generate insights for moderately complex PFT models. Many process models of plankton ecosystems in the oceans, both simple and complex $[13$, $22,49,51]$, represent the interactions of populations $u_{i}$ using equations of the general form:

$$
\dot{u}_{i}=u_{i} f_{i}\left(u_{1}, u_{2}, \ldots, u_{n}\right), \quad i=1, \ldots, n .
$$

Here $\dot{u}=\frac{d u}{d t}$ for $t>0$, and the functions $f_{i}$ are bounded and continuously differentiable in the variables $u_{i}$. The $f_{i}$ describe the gains and losses of each species, trophic guild or PFT, that is $f_{i}=(\text { growth }- \text { predation }- \text { mortality })_{i}$. These $f_{i}$ are often nonlinear functions of the $u_{i}$ and include several parameters that describe the attributes (traits) of the plankton and how they interact. There are many options for the $f_{i}$, and much of the debate surrounding the application of DGOMs centres on whether the forms of the $f_{i}$ and the values of the parameters that distinguish one PFT from another are sufficiently well understood.

We look at the particular case of a model that exactly conserves the mass of limiting nutrient as many models used in biological oceanography also have this property $[22,49,51]$. Many computer simulation models also approximately conserve the total quantity of the key limiting nutrients, and hence run in an approximately closed manner. Nutrient conservation is consistent with the view that export production in 
the ocean is generally balanced by new production $[18,46]$, and that plankton ecosystems approximately conserve mass over annual cycles. Conservation of mass of the key limiting nutrient is one of two formal requirements for a biogeochemical model to be written in "conservative" $(C)$ "normal" $(N)$ form $[11,12]$; these CN conditions ensure ecological verisimilitude, and as a bonus facilitate mathematical analysis.

Impacts of climate change, such as increasing ocean temperature and acidification, on marine plankton systems remain not well understood in 2014 [2, 20, 53]. Changes in plankton populations are controlled by the balances between growth and loss processes, and changes in mortality parameters are often used to capture the essence of this. For instance, Fussmann and Heber [23] consider the ubiquity of chaotic dynamics in 28 food webs of varying complexity, connectivity and numbers of trophic levels. They conclude that chaos should be rare in models with few trophic levels, and should not appear in models with an average trophic level (ATL) less than 1.5. Fussmann and Heber [23] define ATL as:

$$
A T L:=\frac{\sum_{i=1}^{N_{\text {spec }}}\left(1+\bar{\chi}_{i}\right)}{N_{\text {spec }}},
$$

where $N_{\text {spec }}$ is the number of species in the food web and $\bar{\chi}_{i}$ is the average chain length of all food chains linking species $i=1, \ldots, N_{\text {spec }}$ to the basal species. Conventionally, $1+\bar{\chi}_{i}$ is defined as the "trophic level" of species $i$ in a food web. We consider a model with $A T L=1.5$ and a reduced version with $A T L=1.33$. Even though $A T L$ is a slightly ambiguous metric (as it is not clear what is the "basal species" in a system such as ours that allows mixotrophy between primary producers), our analysis suggests that chaotic behaviour is often present in simple low ATL models.

The theme of this work is to explore the nature of the dynamics of a simple DGOM system, where we focus on the interactions between the populations in isolation from the physics. This enables us to identify the endogenous dynamics of the populations, that are then modified when they interact with the physical forcings usually inherent in DGOMs. A key objective of this work is to raise the issue of the role of the physical model in modifying the population dynamics predicted by a DGOM, and whether this is real or an artefact of the numerical integration scheme. We use the example of chaotic dynamics and its robustness, and the implications this may have for the study of ocean plankton ecosystems. We demonstrate the existence of (and calculate Lyapunov exponents and Kaplan-Yorke dimensions for) robust chaotic behaviour in both of our three- and four-dimensional systems. We also show that the chaotic attractors are robust to the addition of random noise, suggesting that daily (as opposed to seasonal) environmental variability may not preclude the occurrence of chaotic dynamics in real systems, although it may move the system from one basin of attraction to another. Finally, we compare the signal, or the observability, of the 
chaos showing that it varies significantly across the plankton populations, obvious in some, invisible in others.

\section{The Dynamic Green Ocean Model}

The simple, uncoupled DGOM $[8,10]$ we investigate here is composed of five coupled ordinary differential equations for bacteria $B^{\prime}$, zooflagellates $F^{\prime}$, dissolved nitrogen $N^{\prime}$, phytoplankton $P^{\prime}$ and zooplankton $Z^{\prime}$. In addition there is a mass conservation equation constraint $B^{\prime}+F^{\prime}+N^{\prime}+P^{\prime}+Z^{\prime}=N_{T}$. These variables are re-scaled by dividing each by the total nutrient $N_{T}: V=V^{\prime} / N_{T}$, for $B^{\prime}, F^{\prime}, N^{\prime}, P^{\prime}$ and $Z^{\prime}$ so that $B+F+N+P+Z=1$. Because of this constraint, we have a four degree of freedom system in $B, F, P, Z$, and determine $N$ from the mass conservation constraint (where $0 \leq B, F, N, P, Z \leq 1$ ).

Cropp and Norbury $[8,10]$ provide a detailed analysis of the critical points and the eigenvalues and eigenvectors that control the dynamics of this $B F(N) P Z$ model, and describe its complicated limit cycles. We extend this investigation to examine the nature and robustness of the chaotic dynamics in the presence of environmental noise affecting the system. The four-dimensional $B F(N) P Z$ model, non-dimensionalised (or scaled) by the measured parameter values given in Table 1 of [10] is:

$$
\begin{aligned}
\dot{B} & =\left(1-k_{11}\right)\left(G_{1}(P, B)+G_{2}(N, B)\right)-G_{3}(B, F)-k_{10} B, \\
\dot{F} & =\left(1-k_{14}\right) G_{3}(B, F)-k_{13} F \\
\dot{P} & =G_{4}(N, P)-G_{1}(P, B)-k_{4} P Z, \\
\dot{Z} & =k_{4}\left(1-k_{20}\right) P Z-k_{19} Z,
\end{aligned}
$$

together with

$$
\begin{aligned}
\dot{N}= & k_{10} B+\left(k_{11}-1\right) G_{2}(N, B)+k_{11} G_{1}(P, B)+k_{14} G_{3}(B, F) \\
& +k_{13} F+k_{19} Z+k_{4} k_{20} P Z-G_{4}(N, P),
\end{aligned}
$$

where we have introduced the Michaelis-Menten / Holling Type II nutrient uptake / grazing interactions

$$
\begin{aligned}
G_{1}(P, B) & =k_{1} B \frac{P}{P+k_{2}}, \\
G_{2}(N, B) & =k_{25} B \frac{N}{N+k_{26}}, \\
G_{3}(B, F) & =k_{8} F \frac{B}{B+k_{9}}, \\
G_{4}(N, P) & =k_{23} P \frac{N}{N+k_{24}}, \\
N & =1-(B+F+P+Z) .
\end{aligned}
$$


Note that $\dot{N}>0$ when $N=0$, which satisfies the CN consistency Lid criterion that constrains the system dynamics to the ecospace $E=[0 \leq B, F, N, P, Z \leq 1]$ (see [11]) and gives physical meaning to $N$. We scale the equations (2.1), with time non-dimensionalised by using the maximum phytoplankton growth rate $\left(k_{23}^{\prime}=0.27\right.$ $[8,10])$, where the populations are scaled by the total nutrient $N_{T}$. We denote the measured parameter values with a prime (i.e. $k_{m}^{\prime}$ ). After substitution the scalings for $m=1,8,10,13,19,23,25$ are $k_{m}=k_{m}^{\prime} / k_{23}^{\prime}$; the scalings for $m=2,9,24,26$ are $k_{m}=k_{m}^{\prime} / N_{T}$; for $m=11,14,20$ are $k_{m}=k_{m}^{\prime}$ and $k_{4}=k_{4}^{\prime} N_{T} / k_{23}^{\prime}$. Using the values given in Table 1 of [10], we have:

$$
\begin{array}{cccc}
k_{1}=1.148, \quad k_{2}=0.693, \quad k_{4}=1.852, & k_{8}=6.185, & k_{9}=0.182, \\
k_{10}=0.259, \quad k_{11}=0.63, \quad k_{13}=0.185, & k_{14}=0.65, & k_{19}=0.185, \\
k_{20}=0.4, \quad k_{23}=1, \quad k_{24}=0.252, & k_{25}=1.148, & k_{26}=0.069 .
\end{array}
$$

In (2.1) $k_{10}, k_{13}$ and $k_{19}$ are the (scaled) coefficients for the mortality rates of bacteria $B$, zooflagellates $F$ and zooplankton $Z$ respectively. The scaling means that these rates are expressed as a fraction of the maximum phytoplankton $P$ growth rate $k_{23}$ which has been set to one. The model does not include a natural mortality rate for phytoplankton as these losses are very small, essentially insignificant compared to grazing and other losses [43]. Cropp and Norbury [8] present linear stability analyses, examples of phase portraits and a schematic of the dynamical behaviour as evidence that varying three of the parameters $k_{10}, k_{13}$ and $k_{19}$ exhibit interesting ranges of the system's behaviour.

\section{Methods}

We take the standard approach of investigating the ubiquity of chaotic dynamics in population models; that is we vary key parameters and synthesise the dynamics of the system into bifurcation transition sequences. These are very compact graphical representations of the dynamics, often presented as the location of equilibrium points and/or the maximum and/or minimum values of oscillations. We focus upon complex oscillatory dynamics and present and discuss bifurcation transition sequences in terms of the local maximum values $B_{\max }, P_{\max }, Z_{\max }$ of $B, P, Z$ that occur as the populations fluctuate, as the mortality parameters $k_{13}$ and $k_{19}$ vary. We use mortality parameters to generate the bifurcations, as is common in these investigations, but note that it is the balance between growth and loss that is the important consideration. The use of mortality parameters is the most transparent and efficacious way of varying this balance; they are not special in any other context. The mortality parameters $k_{13}$ and $k_{19}$ were varied over wide ranges that captured all the "interesting" dynamics but we show only the most important ranges (see figure captions). Variations in the other 
mortality parameter, $k_{10}$, are not shown as this parameter does not generate any new bifurcation transition sequences or chaos.

To produce the diagrams, the parameter ranges above were divided into steps of 0.01 or 0.001 and, for each parameter value, the system (2.1) was integrated. The figures were constructed from integrations over 1,000-6,000 (non-dimensional) time units after ignoring transients. The local maximum values of each variable over each of its oscillatory cycles was plotted - the final state of one integration was used as the initial condition for the next. This ultimately yields a plot of the local maximum amplitudes of each oscillatory solution, plotted as a function of the bifurcation parameter. For each parameter value, single points indicate simple period-one limit cycles, multiple points with a clear structure indicate limit cycles with higher periods, and apparently random points without any obvious structure indicate chaos. The initial condition for $Z$ was modified for some integrations and alternately set to $Z_{\text {initial }} \approx 10^{-304}$ (the smallest positive number representable in most computer architectures) or $Z=0$ to highlight the importance of the accuracy of the integration schemes. We computed the Lyapunov exponents (but only report the leading (maximum) exponent) and Kaplan-Yorke dimensions for representative parameter values to confirm the presence of chaotic dynamics. We refer to the full $B F(N) P Z$ model, where the initial condition for $Z$ may be as small as $10^{-304}$ as the four-dimensional (4-D) model and the reduced model, with $Z=0$ as the $B F(N) P$ or $3-\mathrm{D}$ model.

We assessed the robustness of the attractors of the model to external perturbations, as might be expected to occur in a naturally varying ocean environment, by adding random noise $\mathcal{N}(0,0.01)$ (normally distributed noise with mean zero and variance 0.01 ) to each life function of the model equations:

$$
\dot{u}_{i}=u_{i}\left[f_{i}\left(u_{1}, u_{2}, \ldots, u_{n}\right)+\mathcal{N}(0,0.01)\right], \quad i=1, \ldots, n .
$$

The noise is added in such a way that conservation (2.3e) always holds to machine accuracy. The addition of noise in this manner captures the net effects of environmental fluctuations in light, temperature and nutrient regime, and its impacts on growth and loss rates. The relative level of noise is substantial in a mathematical sense as the magnitudes of the right hand sides of equations (2.1) are typically of the order of 0.001. To put the magnitude of the random numerical perturbations into the context of a real plankton system, $95 \%$ of our numerical perturbations exceed the perturbation to the system that would be caused by changes in the growth rate of phytoplankton if the average daily sea-surface irradiance halved from one day to the next. In contrast to the general principle suggested by the results of Fussmann and Heber [23], we find robust chaotic dynamics in quite generic low trophic order ecological models, even with these strong random environmental forcings. 

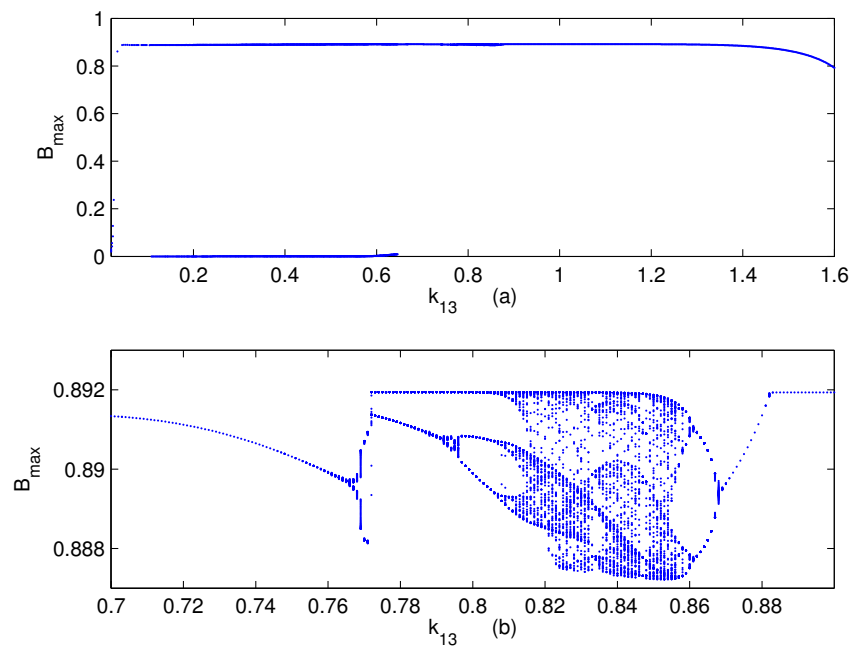

Figure 1: Bifurcation transition diagrams of $B_{\max }$ as $k_{13}$ increases for (a) $0<k_{13}<1.6$ and (b) a blow-up of the region $0.7<k_{13}<0.9$ where chaotic solutions occur. The chaotic dynamics are evident in (b) as the cloud of dots. Chaos is "invisible" in (a) and would not be measurable in practical observations of $B_{\max }$. The maximum Lyapunov exponent of this 4-D system for this parameter set with $k_{13}=0.84$, the middle of the chaotic region, is 0.0118 and the Kaplan-Yorke dimension is 2.25 , confirming the dynamics are chaotic.

\section{Bifurcation transition curves}

\subsection{Bifurcation transition curves as $k_{13}$ varies}

\subsubsection{The 4-D model}

Previous work [8] has shown that varying $k_{13}$ produces the longest range of nontrivial oscillatory dynamics for the $B F(N) P Z$ model. Here, we present bifurcation transition diagrams in terms of the local maximum values of $B, P, Z$ as functions of $k_{13}$ increasing in Figures 1-3.

Figure 1 shows the bifurcation transition diagram of $B_{\max }$ for $0<k_{13}<1.756$, where we lose the $B F(N) P Z$ oscillatory solution at $k_{13}=1.756$ to a stable steady $B F(N)$ state. Not shown here are the integrations to $k_{13}=2$ confirming these results. For $k_{13}>1.814, F<\approx 10^{-(20)}$ and we recover the $B(N)$ stable state. Shown in Figure 1(b) (lower panel) is a blow-up of the parameter window of chaos, which we found for $0.7<k_{13}<0.89$, and $0.887<B_{\max }<0.892$. For $k_{13}<0.892$, the dynamics is governed by the full $B F(N) P Z$ model. For $k_{13}>0.892, Z<\approx 10^{-(20)}$ and the dynamics is now determined by the reduced $B F(N) P$ subsystem whose behaviour will be studied below. This region of chaos is not evident in the bifurcation transition plot of $F_{\max }$ as a function of $k_{13}$, and hence is not shown. It is, however, very apparent 

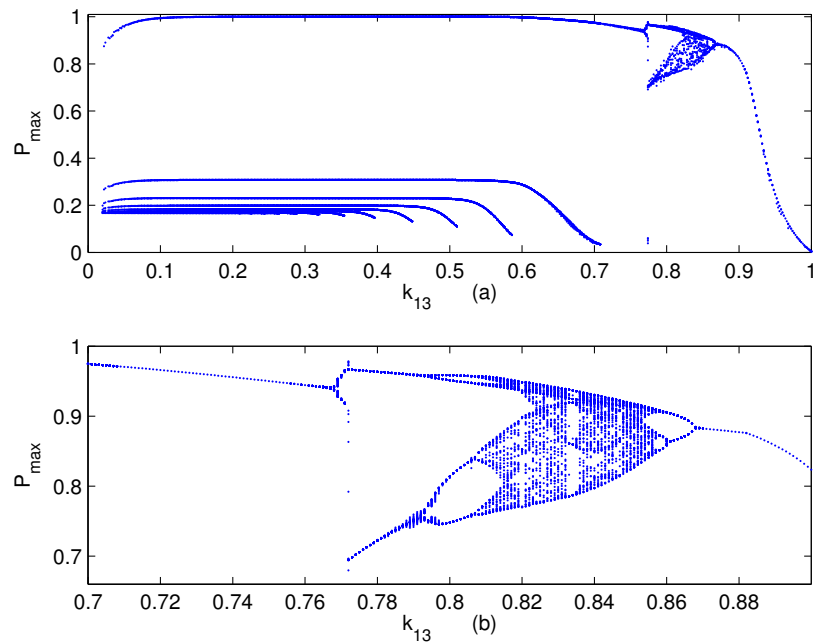

Figure 2: Bifurcation transition diagrams of $P_{\max }$ as $k_{13}$ increases for (a) $0<k_{13}<1$ and (b) a blow-up of the region $0.7<k_{13}<0.9$ showing chaos in the approximate region $0.77<k_{13}<0.87$. Note in contrast to $B_{\max }$ in Figure 1, chaos in $P_{\max }$ is clearly evident, but might not be detectable in systems that include environmental perturbations.

in the corresponding plot for $P_{\max }$ shown in Figure 2, where the region of chaos lies in $0.7<P_{\max }<1$ (lower panel). For $k_{13}>1.01, P \rightarrow 0$ and we have the reduced $B F(N)$ system. Finally Figure 3 shows the bifurcation transition diagram for $Z_{\max }$ for $0.1<k_{13}<0.892$. Again, the region of chaotic behaviour is clearly visible in the range $0<Z_{\max }<0.6$.

We note that if these chaotic dynamics were present in a real system or DGOM model the chaotic behaviour would be experimentally visible if we monitored $P$ or $Z$, but if we monitored $B$ or $F$ our conclusion would be that no chaos was present. Further, note that large grid partial differential equation simulation packages usually do not find solutions or simulate processes to better than $1 \%$ accuracy.

In all of the above we increased $k_{13}$. Next we decreased $k_{13}$ from values where $Z \approx 10^{-(304)}$, approaching the smallest real number representable in double precision computer architecture. We performed two experiments, first, taking initial conditions, appropriate for $k_{13}=1$, but with $Z \approx 10^{-(304)}$, and then setting $Z=0$. The difference between $Z=0$ and $Z \approx 10^{-}$(304) is of no consequence to coupled DGOM predictions of populations, as these models are unable to resolve such differences, however, the analytical implications are clear. $Z=0$ defines a three-population $B F(N) P$ system to which $Z$ non-zero introduces an additional, potentially destabilising, eigenvalue at each critical point, fundamentally changing the properties of the system. The 

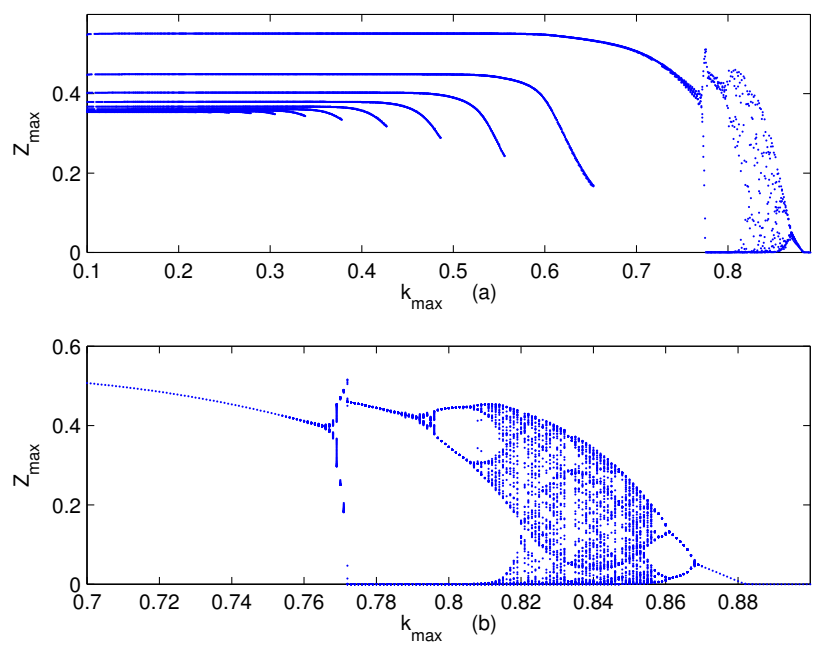

Figure 3: Bifurcation transition diagrams of $Z_{\max }$ as $k_{13}$ increases for (a) $0.1<k_{13}<0.89$ and (b) a blow-up of the region $0.7<k_{13}<0.9$ showing a more detailed view of the chaotic dynamics. In contrast to $B_{\max }$ in Figure 1, chaos in $Z_{\max }$ spans a large amplitude and is clearly evident.

dynamics of the system may not change dramatically as a result, but these simulations reveal that discernible differences are introduced, even at the smallest population size representable in a computer. These differences could not be preserved by the numerical pde solvers used in global coupled DGOMs.

When $k_{13}$ was increased, the local maximum value $Z_{\max }$ reduced until $Z_{\max } \approx$ $10^{-(304)}$ when $k_{13} \approx 0.91$. However, in practical DGOM calculations even effects of the order of $0.1 \%$ are often lost in the "noise", so that any relatively small scale effects would not be evident in the system. This might be interpreted from numerical experiments in the vein that "environmental variations suppress chaos". Figure 4 shows a section of the time series for $P$ and $Z$, and phase portraits in the $(B, P)$ and $(B, F, P)$ spaces for $k_{13}=0.84$. As was apparent from the bifurcation transition diagrams, the chaotic dynamics is compressed in the projection into the $(B, F)$ plane, but is clearly visible in both the $P$ and $Z$ variation and the $(B, P)$ phase plane.

\subsubsection{A reduced 3-D model}

The $B F(N) P Z$ model may be deconstructed into two subsystems, a $B F(N) P$ subsystem and a $B(N) P Z$ subsystem, that each retain a part of the original attractor [10]. Cropp and Norbury [10] showed that the $B(N) P Z$ subsystem has non-chaotic bifurcation behaviour, so we focus our analysis on the $B F(N) P$ subsystem. Here we set $Z=0$ and increase $k_{13}$ from $k_{13}=0.74$ to $k_{13}=1$. We see in Figure 5 that the 

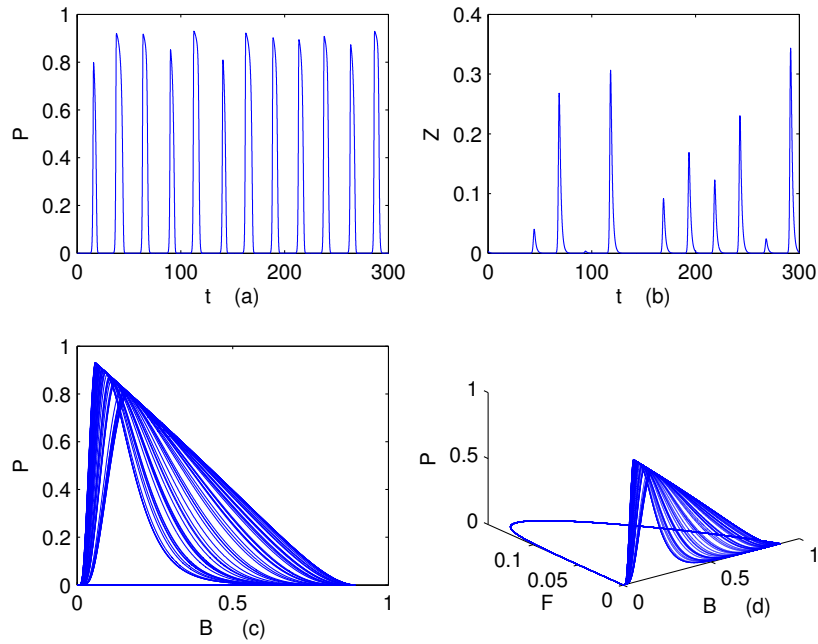

Figure 4: Time series of (a) $P(t)$, (b) $Z(t)$, and phase portraits in the (c) $(B, P)$-plane and (d) the $(B, F, P)$-space for $k_{13}=0.84$ in the 4 -D model. We show the "stiletto" $B-P$ attractor, and the re-normalising (or recovery) loop in $F$. The leading Lyapunov exponent for this case is 0.0118 , with a Kaplan-Yorke dimension of 2.25 .

chaotic region is confined to a much smaller region of $B_{\max }$ parameter space than when $Z \neq 0$. (The vertical scale in the lower panel of Figure 5 is $0.891939<B_{\max }<$ 0.891947.)

Comparison of the plots of $B_{\max }$ for the 4-D system (Figure 1) and for the 3-D system (Figure 5) reveals that the variation in $B_{\max }$ in the chaotic region has been reduced by an order of magnitude by setting $Z=0$. However, comparison of the same plots for $P_{\max }$ (Figures 2 and 6 ) shows that the region of chaotic behaviour of $P_{\max }$ as $k_{13}$ increases is far greater in the 3-D model than in the full 4-D system and extends over the entire range 0-1. Figure 7 shows further comparisons between the dynamics observed in the full 4-D and the reduced 3-D models for a single value of $k_{13}=0.75$. Where we have a stable period-2 cycle in the 4-D system (panels (a),(c) and (e)), we have chaotic behaviour in the simplified/reduced 3-D system (panels (b), (d) and (f)). The period-2 cycle is clearly visible in the $P(t)$ time series and in the projection onto the $(B, P)$ plane, while the chaotic behaviour is clearly visible in both the $P$ and the $B$ time series, as well as in the $(B, P)$ plane projection. In the phase portraits, we have off-set the coordinate axes to show how close the trajectories pass to these axes.

While Figure 1 showed that the 4 -D system is chaotic for $k_{13}=0.84$, Figure 6 shows that in the 3 -D system we find a simple periodic cycle for $k_{13}=0.84$, and a period-2 cycle for $k_{13}=0.82$. The rubric that we draw from this example is 

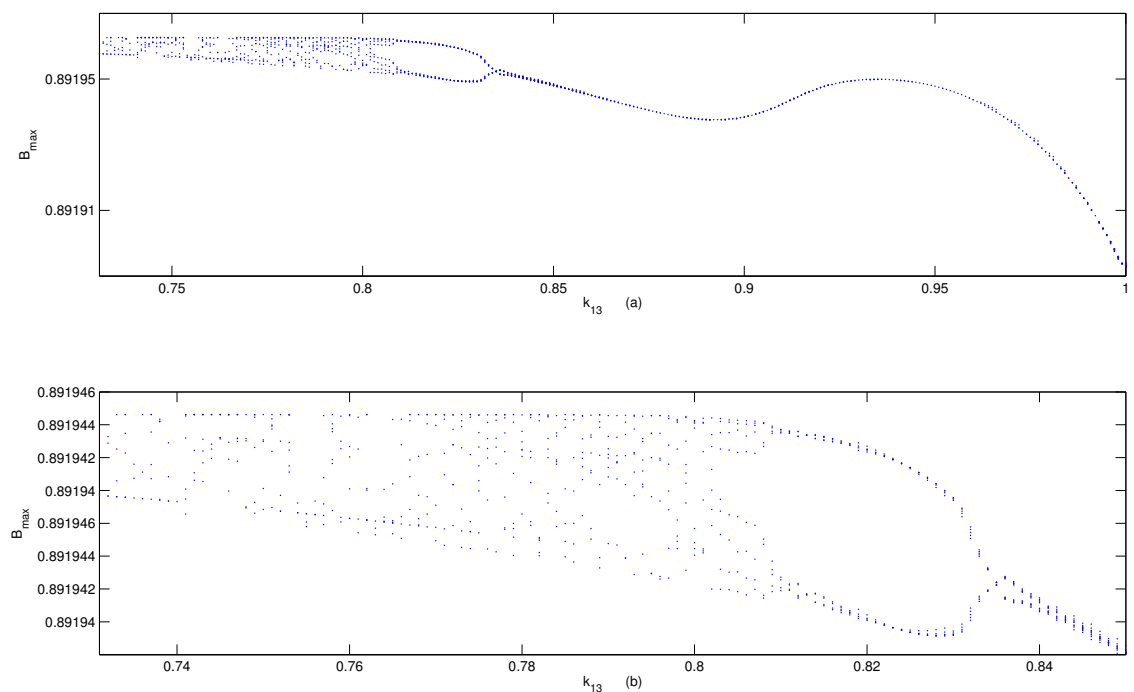

Figure 5: Bifurcation transition diagrams of $B_{\max }$ for the 3-D $B F(N) P$ model as $k_{13}$ increases from $k_{13}=0.74$ to $k_{13}=1$ with $Z=0$. In (a) $0.89191<B_{\max }<0.89195$, and in (b) $0.891939<B_{\max }<$ 0.891947. This level of chaos would be experimentally indistinguishable from regular behaviour. 

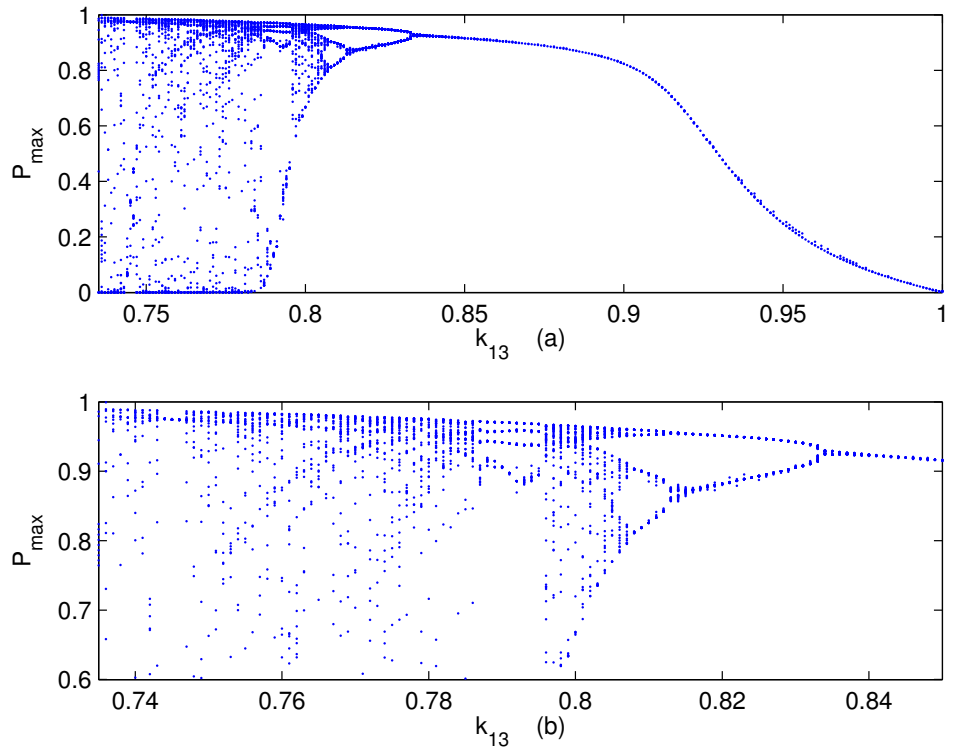

Figure 6: Bifurcation transition diagrams of $P_{\max }$ for the 3-D $B F(N) P$ model as $k_{13}$ increases from $k_{13}=0.74$ to $k_{13}=1$ with $Z=0$. In (a) $0<P_{\max }<1$, while (b) shows a blow-up of the chaotic region, but restricting $P_{\max }>0.6$. In contrast to Figure 5, this chaotic signature is readily observable. 

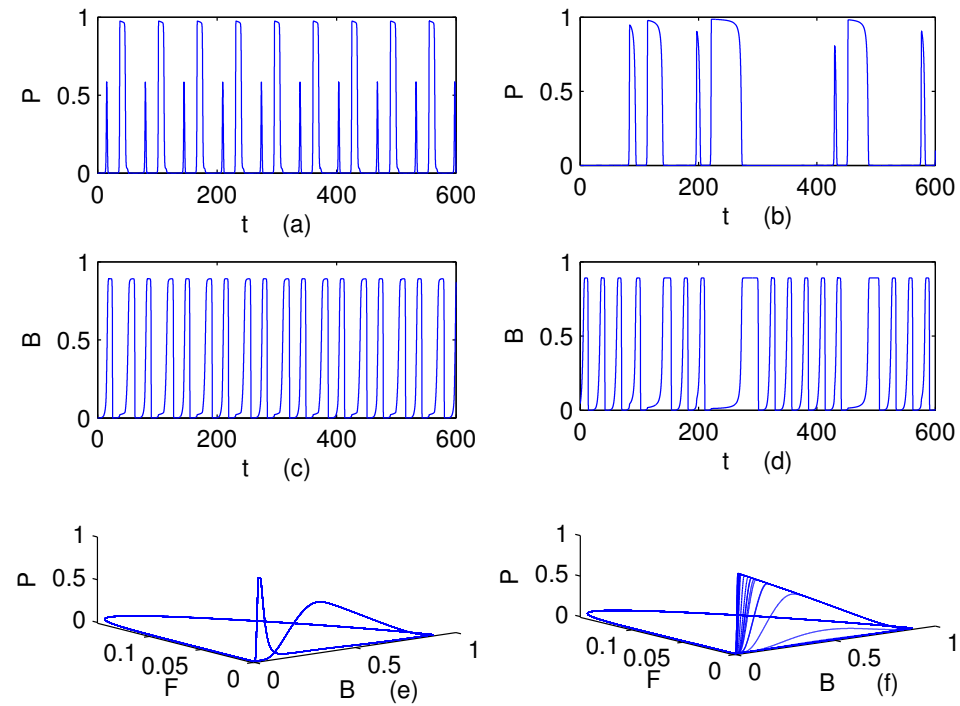

Figure 7: We show time series and phase portraits at $k_{13}=0.75$ for the 4 -D $B F(N) P Z$ system in the left column ((a) $P$, (c) $B$, (e) $(B, F, P)$ phase space), and for the reduced 3-D $B F(N) P$ system in the right column ((b) $P$, (d) $B,(\mathrm{f})(B, F, P)$ phase space). The 4 -D system shows a period-2 limit cycle, while the 3 -D system shows chaos in the timing, size and length of occurrence of the phytoplankton $P$ and bacteria $B$ blooms. The leading Lyapunov exponent of the system for this parameter set with $k_{13}=0.75$, the middle of the chaotic region, is 0.0975 and the Kaplan-Yorke dimension is 2.014 , confirming the dynamics are chaotic. 

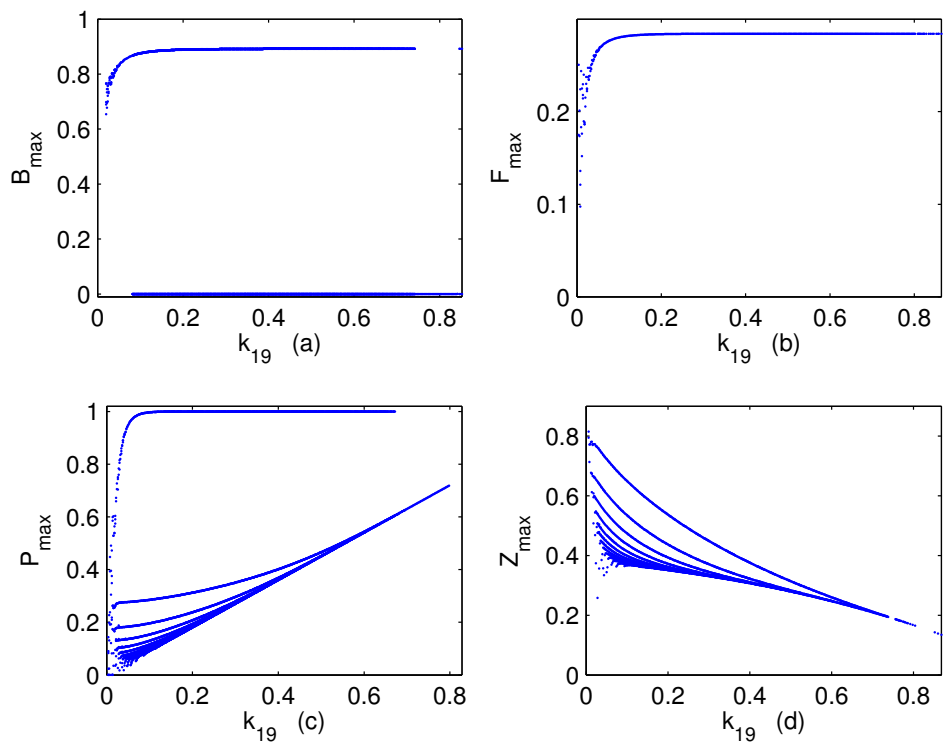

Figure 8: Bifurcation transition diagrams for (a) $B_{\max }$, (b) $F_{\max }$, (c) $P_{\max }$ and (d) $Z_{\max }$ as a function of $k_{19}$ in the 4 -D system.

that simplification of the model ecology (here by setting the initial condition $Z=0$ and consequently ignoring one PFT) does not always simplify the system behaviour. Hence, full spatial simulation models may yield different results on different machines.

\subsection{Bifurcation transition curves when $k_{19}$ varies}

When $k_{19}$ is varied in the 4-D model, with the remaining parameters given by (2.4), we obtain the bifurcation transition plots shown in Figures 8-9. Here, chaotic dynamics are evident only for small values of zooplankton mortality $\left(0<k_{19}<0.05\right)$. The chaotic region is evident in Figure 9, where we plot a blow-up of the bifurcation diagram for $B_{\max }$ (Figure 8) for $0<k_{19}<0.05$. In contrast to $\S 4.1$, the region of chaos exists only for small values of $k_{19}$, albeit for $0<B_{\max }<0.85$. For $k_{19}<0.0005$, we found that $P \approx 10^{-(304)}, Z$ decreases to small values, and $B$ and $F$ undergo periodic oscillations.

Figure 10 shows the behaviour of $B, N$ and $Z$ when $k_{19}=0.001$. The dynamics in the $(B, F)$ plane is chaotic. Figure 10 (a and $\mathrm{b}$ ) reveals interesting behaviour that might be described as chaos with apparent memory [8] . Here, the bacteria population $B$ appears to be perturbed from its trajectory but recovers, "remembers" what it was doing prior to the perturbation, and then continues on the original trajectory until the next perturbation. These perturbations are not evident in the $Z$ population (Figure 


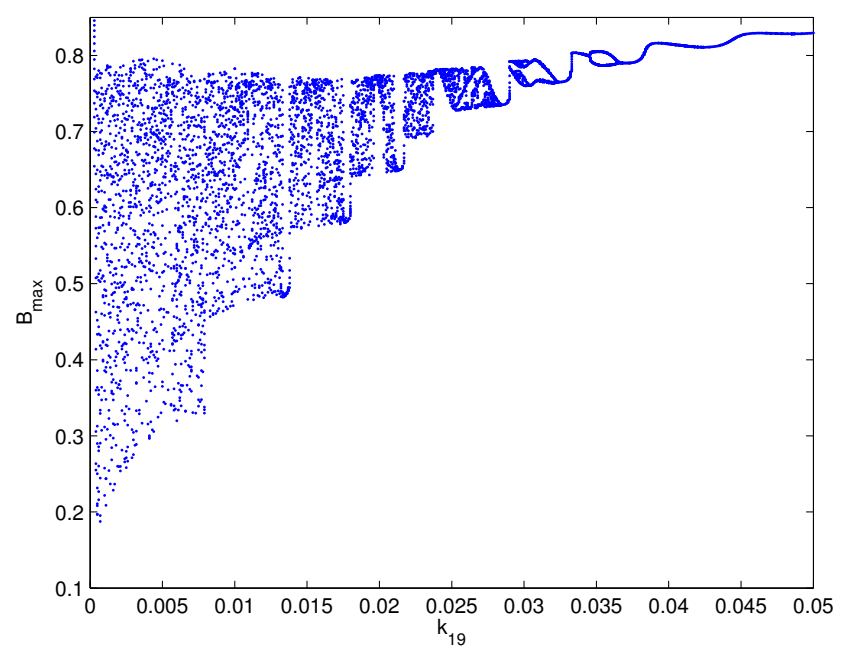

Figure 9: A magnification of the bifurcation transition diagram for $B_{\max }$ for $0<k_{19}<0.05$ showing chaotic dynamics. The leading Lyapunov exponents are given in Table 2.

10). Of course the perturbations are not perturbations in the usual (externally forced) sense, but are an intrinsic part of the endogenous behaviour of the model. Again we make the point that observation of such behaviour in a coupled DGOM would tempt an observer to assume that the physics was perturbing the ecological system. Inspection of a very accurate dynamical system model reveals that such a deduction would be incorrect.

\subsection{Summary}

The bifurcations and paths into and out of chaos shown in Figures 1-10 are but a small sample of the potential for chaos in the $B F(N) P Z$ system, a relatively simple version of the equations that describe population interactions in DGOMs. Within this simple model, chaos may be present subtly (Figure 1), moderately (Figure 2) or obviously (Figure 3) depending on which population is observed. It may appear over large parameter ranges, as for variations of $k_{13}$ in Figures 1-6, or be constrained to very small ranges of the parameter as for variations in $k_{19}$ in Figures 8 and 9. Summaries of the dynamics and the key points in transitions into and out of chaos are listed in Tables 1 and 2 .

Table 1 shows that there are subtle differences between the $B F(N) P Z$ system when $Z$ is small and when $Z=0$ (i.e. the between the full 4-D and reduced 3-D systems). While the dynamics for many values of $k_{13}$ are similar, there are differences in dynamics at some parameter values. Table 2 summarises some key points in the 

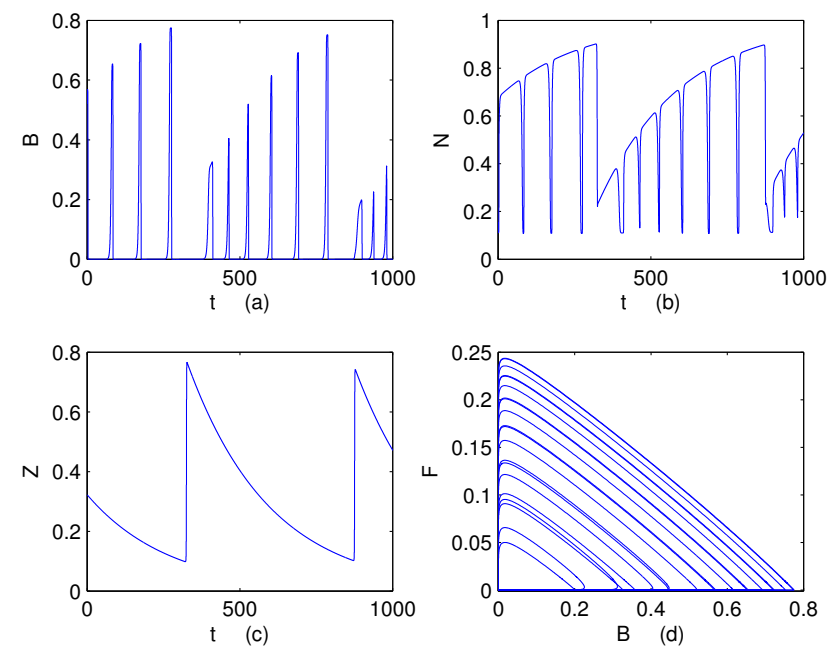

Figure 10: Time series of (a) $B$, (b) $N$ and (c) $Z$ for $k_{19}=0.001$. The $(B, F)$ plane (d) shows the system chaotically resetting itself. The "memory" effect is apparent in (a) and (b) as the recovery of the $B$ population and inorganic nutrient $N$ to its previous trajectory after each spike; there is no evidence of a spike in the $Z$ time series. The leading Lyapunov exponent is 0.1558 and the Kaplan-Yorke dimension is 3.85 .

response of the system to variations in $k_{19}$, for which chaotic dynamics only occur at very small parameter values. Unlike $k_{13}, k_{19}$ does not generate chaos over a large range, but as Figure 9 shows, generates dramatic change when quite small.

The robustness of the attractors considered in Figures $4(\mathrm{c}, \mathrm{d})$ and $7(\mathrm{e}, \mathrm{f})$ is examined in Section 5 .

\section{Robustness of the chaotic attractors}

The robustness of model solutions is a contentious issue. There have been many examples in the literature where models with very particular or contrived properties have been used to demonstrate a point. An example relevant to this discussion is the paper of Huismann and Weissing [29], where the chaotic solutions could be collapsed by varying a single parameter by $2 \%$ [47]. Figure 11 reveals that the added noise has an easily noticeable effect on both the $P$ and $Z$ populations in the spiral part of the limit cycle, where predator-prey interaction $(P Z)$ dominates the $B F(N) P Z$ dynamics; and in the transition from the $P Z$ plane to the $B F$ plane of the limit cycle, where the $B P$ predator-prey interaction dominates the dynamics. The noise has a less noticeable effect in the $B F$ plane of the limit cycle where the predator-prey interaction between $B$ and $F$ dominates the dynamics. 
Table 1: Summary of bifurcation transition features for figures in Section 4 for variations in $k_{13}$.

\begin{tabular}{|c|c|c|c|}
\hline$k_{13}$ & $B F(N) P Z$ & $B F(N) P$ & Comments \\
\hline 0.70 & Period-2 solution & Fixed point at $P=1$ & $\begin{array}{l}\text { The } 4-\mathrm{D} \text { model has a pe- } \\
\text { riodic solution but the } 3- \\
\mathrm{D} \text { model has a fixed point } \\
\text { (Figs } 5,6 \text { ) }\end{array}$ \\
\hline 0.75 & Period-2 solution & $\begin{array}{lll}\text { Chaos } & (L L E= \\
0.0975, & K Y D= \\
2.014) & \end{array}$ & $\begin{array}{l}\text { The } 4 \text {-D model has a pe- } \\
\text { riodic solution but the } 3-\mathrm{D} \\
\text { model has chaos (Fig } 7 \text { ) }\end{array}$ \\
\hline 0.80 & $\begin{array}{l}\text { Chaos }(L L E= \\
0.038, K Y D=2.84)\end{array}$ & $\begin{array}{l}\text { Chaos }(L L E= \\
0.030, K Y D=2.002)\end{array}$ & $\begin{array}{l}\text { Both the } 3-\mathrm{D} \text { and } 4-\mathrm{D} \text { mod- } \\
\text { els have chaotic dynamics }\end{array}$ \\
\hline 0.84 & $\begin{array}{l}\text { Chaos }(L L E=0.012, \\
K Y D=2.25)\end{array}$ & Period-1 solution & $\begin{array}{l}\text { The } 4-\mathrm{D} \text { and } 3-\mathrm{D} \text { models } \\
\text { have similar chaotic solu- } \\
\text { tions }\end{array}$ \\
\hline 0.90 & Period-1 solution & Period-1 solution & $\begin{array}{l}\text { The models have a similar } \\
\text { periodic solution }\end{array}$ \\
\hline
\end{tabular}

Note: LLE refers to the Leading Lyapunov Exponent, where a positive value indicates chaotic dynamics (all Lyapunov Exponents are negative for systems without chaos). KYD refers to the Kaplan-Yorke dimension, where a fractional value is associated with chaotic dynamics.

Table 2: Summary of bifurcation transition features for figures in Section 4 for variations in $k_{19}$.

\begin{tabular}{|c|c|c|c|}
\hline$k_{19}$ & Dynamics & & Comments \\
\hline 0.0005 & Period-1 solution & & Limit cycle involving $B$ and $F$ only \\
\hline 0.001 & $\begin{array}{l}\text { Chaos } \quad(L L E \\
0.159, K Y D=3.85\end{array}$ & $=$ & Chaotic solutions clearly evident in $B_{\max }$ \\
\hline 0.02 & $\begin{array}{l}\text { Chaos } \quad(L L E \\
0.034, K Y D=3.48\end{array}$ & $=$ & Chaotic solutions with reducing amplitude \\
\hline 0.05 & Period-1 solution & & $\begin{array}{l}\text { Large amplitude population oscillations in } \\
\text { full system }\end{array}$ \\
\hline 1.11 & Fixed point & & Equilibrium solution at $P=1[8]$ \\
\hline
\end{tabular}



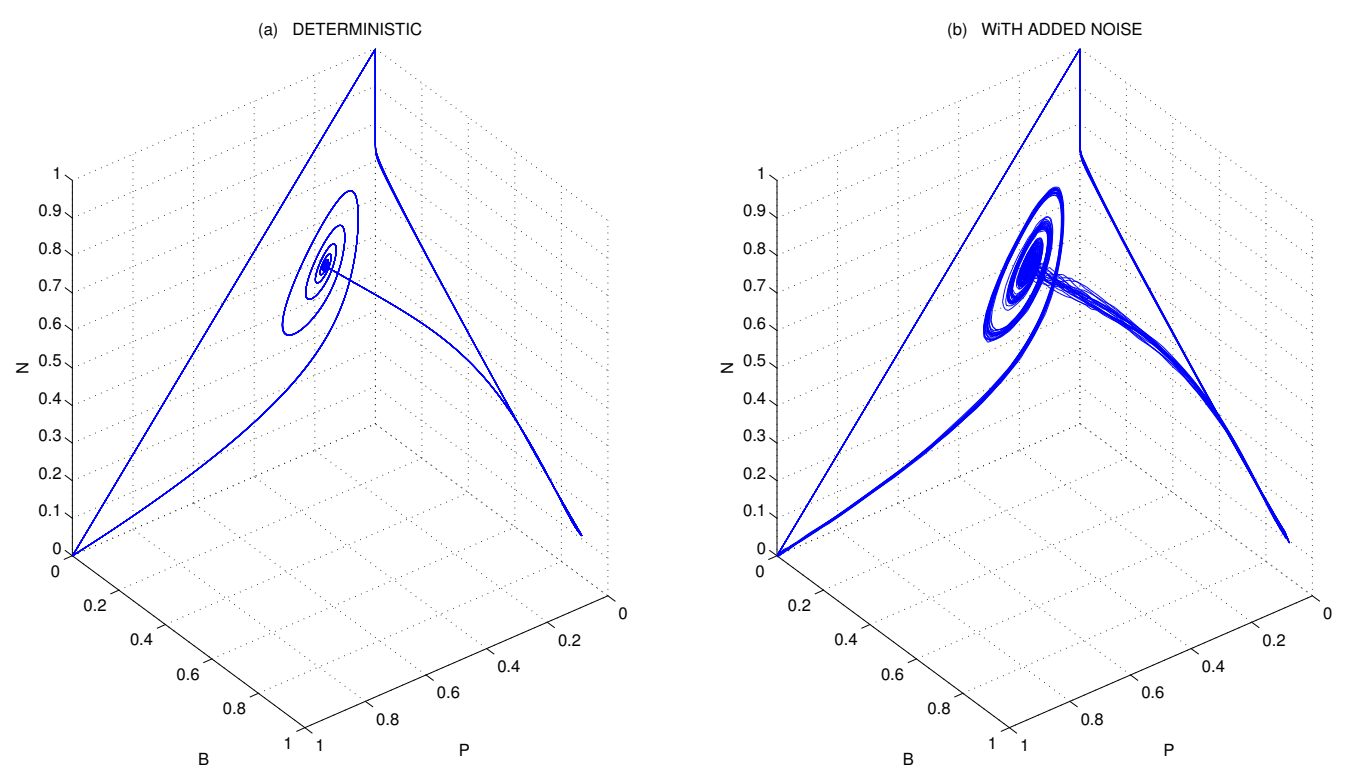

Figure 11: The attractor of the $B F(N) P Z$ system for the default parameter values (2.4) (left panel), and with added noise (right panel), both shown in $P B N$ space. $N$ acts as a surrogate for $Z$ dynamics in the $(N) P$ plane and as a surrogate for $F$ dynamics in the $B(N)$ plane. The added noise is normally distributed with a mean of zero and variance 0.01 . 

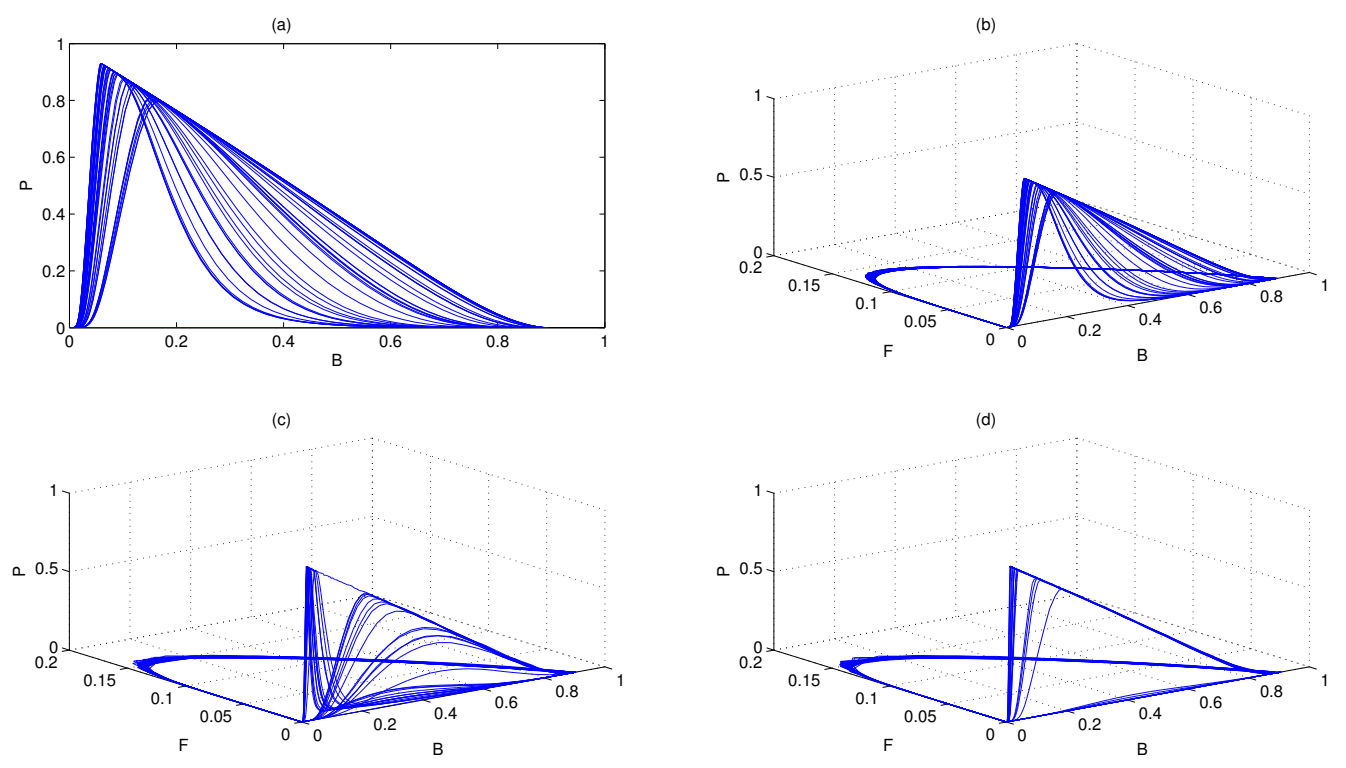

Figure 12: The chaotic attractors identified previously while varying $k_{13}$ are reproduced with added noise $\mathcal{N}(0,0.01)$ demonstrating their robustness: (a) Figure 4(c), (b) Figure 4(d), (c) Figure 7(e) and (d) Figure 7(f), all with noise added. $k_{13}=0.84$ in (a), (b) and $k_{13}=0.75$ in (c), (d). Note the recovery in the $B F$ plane in (b), (c) and (d) is regular compared to that in Figure 10(d). Recall that (a-c) are dynamics of the 4-D model and (d) is dynamics of the 3-D model.

The chaotic attractors previously identified while varying $k_{13}$ in Figures 4 and 7 turn out to be robust to the relatively large level of noise added to the system, as shown by Figure 12. In each case the effect of the noise is easily noticeable, but the full $B F(N) P Z$ and reduced $B F(N) P$ systems maintain their chaotic orbits. The $B F(N) P$ system has a separatrix that separates the chaotic attractor, which closely visits an unstable point at $P=0.9894$, from a nearby stable point at $P=1$. Increasing the noise added to the system by an order of magnitude (i.e. mean zero, standard deviation 0.10) causes the system to jump the separatrix and be attracted to the stable point at $P=1$. However, this point is unstable in the full $B F(N) P Z$ system. Hence increasing the noise in the full system by an order of magnitude does not cause the loss of the chaotic attractor, but does noticeably increase its variance.

The chaotic dynamics previously identified while varying $k_{19}$ in Figures 9 and 10 are maintained when a significant level of noise is added to the system. As for the attractors found by varying $k_{13}$, the effect of the added noise is easily noticeable in that orbits are clearly perturbed on the attractors, but the attractors are robust to (relatively) substantial perturbations. 


\section{Discussion}

This work has considered the dynamics of an isolated DGOM, first given in $[8,10]$ and similar to, but simpler than, those being developed for applications in climate change simulation. Our $B F(N) P Z$ model, although less complex than operational PFT models or DGOMs, has an interesting and complicated limit cycle attractor for parameter values that have been measured for plankton in the Earth's oceans. The model also exhibits chaotic dynamics, revealing a new attractor that we characterise as the "stiletto attractor" (see Figure 4 (c), (d)), that can be generated by two bifurcation parameters; $k_{13}$, the zooflagellate mortality rate, and $k_{19}$, the zooplankton mortality rate. It is important to note that there is nothing special in mortality parameters in generating chaos, but that they are often used as the simplest and most direct way of perturbing the system. It is the balance between the growth and death terms in the equations that is key to the behaviours, and it is not unusual for a particular behaviour to be achieved by varying any parameter in a model (for example, see [9]). While mortality parameters will be important for DGOMs that model the impacts of climate change on marine systems, as mortality rates may be sensitive to prolonged temperature variations, they should not be the only focus as changes in growth rates and/or grazing rates may have similar sensitivities and impacts.

The chaotic dynamics persist even when we reduce the dimension of the model to the minimum possible to have chaos (which is three populations). The addition of Gaussian white noise does not significantly affect the chaotic attractors of the $B F(N) P Z$ model, suggesting that chaos is a relatively robust feature of this model. It may, however, significantly affect the dynamics of the reduced $B F(N) P$ system as large environmental perturbations can cause the system to 'jump' a separatrix that separates the basin of attraction of the chaotic attractor from that of a stable critical point. Our simulations with very substantial relative levels of random noise added to these systems, representing the environmental perturbations that all ecosystems experience, reveal that the chaotic attractors we present are quite robust, suggesting that these results are not attributable to a sensitive mathematical structure or a delicate parameter set. It also suggests that environmental variations at daily time scales are unlikely to be stabilising mechanisms, nor the reason that chaotic dynamics are rarely observed in real ecosystems. Our results also suggest that low dimensional ecosystem models, even smooth models with only one or two trophic levels may not have as straightforward dynamical behaviour as is often reported in the literature.

Fussmann and Heber [23] make the important point that anthropogenic interventions in ecosystems (or in our context, responses to climate change) are more likely to succeed if the dynamics of the system are understood. Our analyses illuminate this observation, as removing $F$ from the $B F(N) P Z$ system when $k_{13}=0.84$ renders a chaotic system stable, but removing $Z$ from the same system leaves the 
chaotic dynamics relatively unaffected. Further, and somewhat counter-intuitively, if the $B F(N) P$ system with $k_{13}=0.75$ is invaded by $Z$, its chaotic dynamics will be reduced to a simpler and more predictable period-2 limit cycle. We note that while the chaotic behaviour is strongly evident in some populations, it is virtually or effectively invisible in others. Chaos may well then be in the eye of the beholder - if it is present in populations that are not of interest but effectively invisible in key populations, then the consensus may be that the system is stable. This might have important consequences for assessments of how the system might respond to change, as systems bordering on chaos are known to be more sensitive to external perturbation [30].

Our results suggest that chaos in ecosystem models may not be the province of just a few carefully chosen or especially complex models, but may occur in relatively simple, pragmatic systems that realistically simulate three or more populations. The role played by chaotic dynamics in real ecosystems remains uncertain, while anecdotal evidence suggests that chaos is rarely observed when chaotic ecosystem equations are coupled to physical models. Whether this suppression of chaos represents reality or is a result of the numerical scheme used to solve the coupled system is unclear. Similarly we have shown that the ecological equations can generate behaviour that in a coupled DGOM would tempt an observer to assume that the physics was perturbing the ecological system. Until questions such as these are resolved, we suggest that the apparently common rubric, that there is nothing that the analysis of simple models can contribute to the understanding of complex models, should be treated with caution.

\section{Conclusions}

The investigations reported here, while considering only the dynamics of a simple uncoupled DGOM, have some useful implications for the construction and use of operational DGOMs in climate studies and fisheries management. We do not suggest that individual explicit results provide general insights in to the dynamics of the DGOMs, but instead provide useful general heuristics that demonstrate that the analysis of simple models can aid the development of these models. We reiterate some important points in this context. The first is the effect of random perturbations to the system, as real plankton systems are subject to continual environmental perturbations. These include both small and regular perturbations associated with the natural spatial and temporal changes in the oceans, and large and unprecedented perturbations associated with anthropogenic impacts such as the introduction of invasive species, fishing and climate change. The chaotic attractors we observed are quite robust and persist when subjected to the equivalent of large environmental perturbations; however very large perturbations can move the system from one basin of attraction to another, resulting in dramatic changes over short time scales. 
Secondly, we note that our comparison of the dynamics of the full $B F(N) P Z$ system and the reduced $B F(N) P$ system reveal that the effects of local species extinctions (for example via fishing) or introductions (by invasive species) may lead to surprising outcomes for natural resource managers, with extinctions not necessarily reducing the propensity for chaos and vice versa. Similarly, the subtleties of the hysteresis observed when parameters were varied in opposite directions may not be evident and may lead managers to incorrect assumptions about how systems might recover from over-exploitation. Further, we find robust chaotic dynamics in quite generic low trophic order ecological models. However, our results are in general consistent with the rubric of Fussmann and Heber [23] that increasing the complexity of ecosystem models generally increases the propensity for chaos.

Finally, and most importantly, the implications of the effects of perturbations and changes to the ecosystem structure noted above must be considered in the context that DGOMs are implemented as population interaction models coupled to physical models that resolve the spatial dynamics of the ocean in computer simulations. Integrations of these partial differential equation implementations of the DGOMs are unable to achieve the accuracy or precision of integrations possible in the ordinary differential equation version we have investigated above. In DGOM simulations, it is never clear whether it is the endogenous dynamics of the ecosystem equations, the exogenous dynamics imposed by the physics, or the constraints inherent in the numerical scheme used to solve the coupled system, that leads to any particular feature of the model output. There is little theoretical or empirical evidence to assist modellers in deciding whether or not their results reflect real world behaviour. Experimental work by Roelke et al. [44] suggests that chaos can be suppressed by environmental forcings, in mesocosm experiments at least. Our results showing robust chaotic attractors contrast with the sensitivity of chaotic models such as that of Huismann and Weissing [29] to subtle changes in parameter values, as shown by Schippers et al. [47]. Further, we show that if we observe the "wrong" population (say in our case bacteria or zooflagellates), then we may fail to notice chaotic behaviour that is much more visible in the other populations (in this case phytoplankton or zooplankton). Somewhat more seriously, if we use numerical simulations that fail to preserve the "invisible chaos" in one population, this might control the chaos in the remaining populations.

Our work suggests that chaotic dynamics are not necessarily fragile or rare in parameter space and can be robust and resistant to the effects of perturbations, and that chaos may be readily observable in some populations but not in others. The attribution to a particular cause of any ecological dynamics derived from the integration of a coupled DGOM needs to be undertaken with great care. It is essential that the endogenous dynamics of both the physical and biological equations need to be understood in isolation, as the output of a coupled DGOM is the product of these interactions. The relative importance of these interactions must be understood, as 
the magnitude and timing of the responses of each system are likely to be different. While the physical system may well dominate ecosystem responses to climate change over long time scales, ecosystem interactions may well dominate responses over short time scales. The analysis of simple ode models of ecosystems still has much to contribute to attempts to predict the response of marine systems to change. In the more than 35 years following Gilpin [24] the extent to which chaotic behaviour affects real ecosystems remains a fundamental question.

\section{Acknowledgements}

We thank an anonymous reviewer whose thoughtful reviews greatly helped in the preparation of this article.

\section{References}

[1] Thomas R. Anderson. Plankton functional type modelling: running before we can walk? J. Plankton Res., 27(11):1073-1081, 2005.

[2] J. Blackford. Predicting the impacts of ocean acidification: Challenges from an ecosystem perspective. Journal of Marine Systems, 81:12-18, 2010.

[3] L. Bopp, L. Resplandy, J. Orr, S. Doney, J. Dunne, M. Gehlen, P. Halloran, C. Heinze, T. Ilyina, R. Sfrian, J. Tjiputra, and M. Vichi. Multiple stressors of ocean ecosystems in the 21st century: Projections with cmip5 models. Biogeosciences, 10:6225-6245, 2013.

[4] J. Bruggeman and S. A. L. M. Kooijman. A biodiversity-inspired approach to aquatic ecosystem modeling. Limnology and Oceanography, 52:1533-1544, 2007.

[5] R.J. Charleson, J.E. Lovelock, M.O. Andreae, and S.G. Warren. Oceanic phytoplankton, atmospheric sulphur, cloud albedo and climate. Nature, 326:655-661, 1987.

[6] J.R. Clark, T.M. Lenton, H.T.P. Williams, and S.J.. Daines. Environmental selection and resource allocation determine spatial patterns in picophytoplankton cell size. Limnology and Oceanography, 58:1008-1022, 2013.

[7] R. Cropp and J. Norbury. Modelling plankton and the library of lotka. Journal of Marine Systems, 2012.

[8] R.A. Cropp and J. Norbury. Investigations into a plankton population model: mortality and its importance in climate change scenarios. Ecological Modelling, 201(2):97-117, 2007. 
[9] R.A. Cropp and J. Norbury. Parameterizing plankton functional type models: insights from a dynamical systems perspective. Journal of Plankton Research, 31:939-963, 2009.

[10] R.A. Cropp and J. Norbury. Simple predator-prey interactions control dynamics in a plankton foodweb model. Ecological Modelling, 220(13-14):1552-1565, 2009.

[11] R.A. Cropp and J. Norbury. Constructing ecologies. Journal of Theoretical Biology, 294:1-8, 2012.

[12] R.A. Cropp and J. Norbury. The mechanisms of coexistence and competitive exclusion in complex plankton ecosystem models. Ecosystems, 15:200-212, 2012.

[13] R.A. Cropp, J. Norbury, A. J. Gabric, and R. D. Braddock. Modeling dimethylsulphide production in the upper ocean. Global Biogeochemical Cycles, 18(GB3005):doi:10.1029/2003GB002126., 2004.

[14] B. Deng. Food chain chaos due to a junction-fold point. Chaos, 11(3):514-525, 2001.

[15] B. Deng. Food chain chaos with canard explosion. Chaos, 14(4):1083-1092, 2004.

[16] B. Deng and G. Hines. Food chain chaos due to shilnikov's orbit. Chaos, $12(3): 533-538,2002$.

[17] B. Deng and G. Hines. Food chain chaos due to transcritical point. Chaos, $13(2): 578-585,2003$.

[18] R.W. Eppley, E.H. Renger, and W.G. Harrison. Nitrate and phytoplankton production in southern california waters. Limnology and Oceanography, 24:483494, 1979.

[19] P. Falkowski, E.A. Laws, R.T. Barber, and J.T. Murray. Phytoplankton and their role in primary, new and export production. In M.J.R. Fasham, editor, Ocean Biogeochemistry, pages 99-121. Springer, New York, 2003.

[20] K.J. Flynn, D.K. Stoecker, A. Mitra, J.A. Raven, P.M. Glibert, P.J. Hansen, E. Graneli, and J.M. Burkholder. Misuse of the phytoplankton ? zooplankton dichotomy: the need to assign organisms as mixotrophs within plankton functional types. Journal of Plankton Research, 35:3-11, 2013.

[21] M.J. Follows, S. Dutkiewicz, S. Grant, and S.W. Chisholm. Emergent biogeography of microbial communities in a model ocean. Science, 315:1843-1846, 2007. 
[22] P.J.S. Franks. Npz models of plankton dynamics: their construction, coupling to physics, and application. Journal of Oceanography, 58:379-387, 2002.

[23] G.F. Fussmann and G. Heber. Foodweb complexity and chaotic population dynamics. Ecology Letters, 5:394-401, 2002.

[24] M.E. Gilpin. Spiral chaos in a predator-prey model. The American Naturalist, 113:306-308, 1979.

[25] T. Hashioka, M. Vogt, Y. Yamanaka, C. Le Quere, E.T. Buitenhuis, M.N. Aita, S. Alvain, L. Bopp, T. Hirata, I. Lima, S. Sailley, and S.C. Doney. Phytoplankton competition during the spring bloom in four plankton functional type models. Biogeosciences, 10:6833-6850, 2013.

[26] A. Hastings and T. Powell. Chaos in a three species food chain. Ecology, 72:896903, 1991.

[27] J.T. Holt, M. Butenschon, S.L. Wakelin, Y. Artioli, and J.I. Allen. Oceanic controls on the primary production of the northwest european continental shelf: model experiments under recent past conditions and a potential future scenario. Biogeosciences, 9:97-117, 2012.

[28] Raleigh R. Hood, Edward A. Laws, Robert A. Armstrong, Nicholas R. Bates, Christopher W. Brown, Craig A. Carlson, Fei Chai, Scott C. Doney, Paul G. Falkowski, Richard A. Feely, Marjorie A. M. Friedrichs, Michael R. Landry, J. Keith Moore, David M. Nelson, Tammi L. Richardson, Baris Salihoglu, Markus Schartau, Dierdre A. Toole, and Jerry D. Wiggert. Pelagic functional group modeling: Progress, challenges and prospects. Deep Sea Research Part II: Topical Studies in Oceanography, 53(5-7):459-512, 2006.

[29] J. Huismann and F.J. Weissing. Biodiversity of plankton by species oscillation and chaos. Nature, 402:407-410, 1999.

[30] S.A. Kauffman. The Origins of Order: Self-Organisation and Selection in Evolution. Oxford University Press, Oxford, 1993.

[31] Michio J. Kishi, Makoto Kashiwai, Daniel M. Ware, Bernard A. Megrey, David L. Eslinger, Francisco E. Werner, Maki Noguchi-Aita, Tomonori Azumaya, Masahiko Fujii, Shinji Hashimoto, Daji Huang, Hitoshi Iizumi, Yukimasa Ishida, Sukyung Kango, Gennady A. Kantakov, Hyun-cheol Kim, Kosei Komatsu, Vadim V. Navrotsky, S. Lan Smith, Kazuaki Tadokoro, Atsushi Tsuda, Orio Yamamura, Yasuhiro Yamanaka, Katsumi Yokouchi, Naoki Yoshie, Jing Zhang, Yury I. Zuenko, and Vladimir I. Zvalinsky. Nemuroa lower trophic level 
model for the north pacific marine ecosystem. Ecological Modelling, 202:12-25, 2007.

[32] C. Le Quere. Reply to horizons article 'phytoplankton functional type modelling: running before we can walk' anderson 2005: I - abrupt changes in marine ecosystems? Journal of Plankton Research, 28(doi:10.1093/plankt/fbl014):871-872, 2006.

[33] C. Le Quere, S.P. Harrison, I.C. Prentice, E.T. Buitenhuis, O. Aumonts, L. Bopp, H. Claustre, L. Cotrim da Cunha, R.J. Geider, X. Giraud, C. Klaas, K.E. Kohfeld, L. Legrende, M. Manizza, T. Platt, R.B. Rivkin, S. Sathyendranath, J. Uitz, A. Watson, and D. Wolf-Gladrow. Ecosystem dynamics based on plankton functional types for global ocean biogeochemistry models. Global Change Biology, 11(doi:10.1111/j1365-2486.2005.01004.x):2016-2040, 2005.

[34] M. Loreau. From Populations to Ecosystems., volume 46 of Monographs in Population Biology. Princeton University Press, Woodstock, Oxfordshire, 2010.

[35] R.M. May and A. McLean. Theoretical Ecology: Principles and Applications. Oxford University Press, Oxford, 2007.

[36] K.S. McCann and P. Yodzis. Bifurcation structure of a three-species food chain model. Theoretical Population Biology, 48:93-125, 1995.

[37] M. Mitchell. An Introduction to Genetic Algorithms. MIT Press, Cambridge, 1998.

[38] C.L. Moloney and J.G. Field. General allometric equations for rates of nutrient uptake, ingestion and respiration in plankton organisms. Limnology and Oceanography, 34:1290-1299, 1989.

[39] J.K. Moore, S.C. Doney, J.A. Kleypas, D.M. Glover, and I.Y. Fung. An intermediate complexity marine ecosystem model for the global domain. Deep-Sea Research II, 49:403-462, 2002.

[40] J. Pastor. Mathematical Ecology of Populations and Ecosystems. WileyBlackwell, Chichester, 2008.

[41] A. Prowe, E. Friederike, M. Pahlow, S. Dutkiewicz, M. Follows, and A. Oschillies. Top-down control of marine phytoplankton diversity in a global ecosystem model. Progress in Oceanography, 101(1):1-13, 2012.

[42] J. A.; Raven and P. G. Falkowski. Oceanic sinks for atmospheric co2. Plant, Cell and Environment, 22(6):741-755, 1999. 
[43] C. Reynolds. Ecology of Phytoplankton. Cambridge University Press, New York, 2006.

[44] D. Roelke, S. Augustine, and Y. Buyukates. Fundamental predictability in multi species competition: the influence of large disturbance. The American Naturalist, 162(5):615-623, 2003.

[45] S.F. Sailley, M. Vogt, S.C. Doney, M.N. Aita, L. Bopp, E.T. Buitenhuis, T. Hashioka, I. Lima, C. Le Qur, and Y. Yamanaka. Comparing food web structures and dynamics across a suite of global marine ecosystem models. Ecological Modelling, 261-262:43-57, 2013.

[46] J. Sarmiento and N. Gruber. Ocean Biogeochemical Dynamics. Princeton University Press, Princeton, 2006.

[47] P. Schippers, A.M. Verschoor, M. Vos, and W.M. Mooij. Does "supersaturated coexistence" resolve the "paradox of the plankton"?. Ecology Letters, 4:404-407, 2001.

[48] B. Sinha, E. Buitenhuis, C. Le Quere, and T.R. Anderson. Comparison of the emergent behaviour of a complex ecosystem model in two ocean general circulation models. Progress in Oceanography, 84:204-224, 2010.

[49] Y.H. Spitz, J.R. Moisan, and M.R. Abbott. Configuring an ecosystem model using data from the bermuda atlantic time series (bats). Deep-Sea Research II, 48:1733-1768, 2001.

[50] F. Suppe. Understanding scientific theories: an assessment of developments 1969-1998. Philosophy of Science, 67:S102-S115, 2000.

[51] S.M. Vallina, R Simo, E.E. Popova, T.R. Anderson, A. Gabric, R.A. Cropp, and J.M. Pacheco. A dynamic model of ocean sulfur (dmos) applied to the sargasso sea: simulating the dimethylsulfide (dms) summer paradox. Journal of Geophysical Research (Biogeosciences), 113:G01009, doi:10.1029/2007JG000415, 2008.

[52] M. Vancoppenolle, L. Bopp, G. Madec, J. Dunne, T. Ilyina, P.R. Halloran, and N. Steiner. Ocean primary productivity from cmip5 simulations: Uncertain outcome, but consistent mechanisms. Global Biogeochemical Cycles, 27:605-619, 2013.

[53] S. Wilken, J. Huisman, S. Naus-Wiezer, and E. Van Donk. Mixotrophic organisms become more heterotrophic with rising temperature. Ecology Letters, $16: 225-233,2013$. 